\title{
On the Problems of Knowledge Acquisition and Representation of Expert System for Diagnosis of Coronary Artery Disease (CAD)
}

\author{
L. J. Muhammad ${ }^{1}$, E. J. Garba ${ }^{2}$, N. D. Oye ${ }^{3}$ and G. M. Wajiga ${ }^{4}$ \\ ${ }^{1}$ Mathematics and Computer Science Department, Federal University, \\ Kashere, Gombe State \\ ${ }^{2,3,4}$ Computer Science Department, Modibbo Adama University of Technology, \\ Yola \\ Imljtech@gmail.com, 22e.i.garba@mautech.edu.ng, ${ }^{3}$ oyenath@yahoo.co.uk, \\ ${ }^{4}$ gwajiga@gmail.com
}

\begin{abstract}
Expert system is computer software that nearly earns the decision making ability of human expert and it is one of the most promising areas of applied artificial intelligence. It had proved its importance in medical field particularly diagnosis of diseases. Expert system has three fundamental techniques namely, knowledge acquisition, knowledge inference and knowledge representation that involved into transferring expertise from human expert to the expert system then to user. Knowledge acquisition and knowledge presentation have been since termed as the problematic bottlenecks in the construction of the application oriented expert system. In this study, the previous studies carried out by various researchers based on knowledge acquisition and presentation of expert system for diagnosis of coronary artery disease have been reviewed and the weaknesses of each work have been spelt out. These spelt put drawbacks of the studies have termed as a knowledge gap to bridge in subsequent study to carry out.
\end{abstract}

Keywords: Artificial Intelligence, data mining, Fuzzy Logic, Knowledge Base, Support system

\section{Introduction}

Medical diagnosis is a process to detect a disease by measuring its specific signs and symptoms. Signs are observed by a physician while symptoms are expressed by the patient. However, patients may not able to precisely express their feelings on the symptoms and doctors may not always sure about the condition of signs. Therefore, various types of uncertainties may be noticed during the diagnosis process of a disease. These uncertainties should be carefully handled, enabling the accurate diagnosis of a disease. Medical practitioners sometime exhibit variation in decision making because of their approaches to deal with uncertainties and vagueness in the knowledge and information. The diagnostic decisions also depend upon experience, expertise and perception of the practitioner. As the complexity of system increases, it is not easy to follow a particular path of diagnosis without any mistake [28].

It is on this note; nowadays expert systems or knowledge based systems are being used to emulate the decision-making ability of a human medical expert so as to minimize the complexity and uncertainty with regards to diagnosis of diseases. Expert system is a software system that nearly earns the decision making ability of a human expert. The goal of an expert system is to solve a complex problem by reasoning with knowledge rather than conventional procedural codes. Expert systems are the commonest type of Artificial Intelligence systems in routine clinical use. They contain medical knowledge, usually

Received (January 25, 2018), Review Result (May 17, 2018), Accepted (May 29, 2018) 
about a very specifically defined task, and are able to reason with data from individual patients to come up with reasoned conclusions. There are many different types of clinical task to which expert systems are applied.

i. Diagnostic assistance: When a patient's case is complex, rare or the person making the diagnosis is simply inexperienced, an expert system can help come up with likely diagnoses based on patient data.

ii. Therapy critiquing and planning: Systems can either look for inconsistencies, errors and omissions in an existing treatment plan, or can be used to formulate a treatment based upon a patient's specific condition and accepted treatment guidelines.

iii. Image recognition and interpretation: Many medical images can now be automatically interpreted, from plane X-rays through to more complex images like angiograms, CT and MRI scans. This is of value in mass-screenings, for instance, when the system can flag potentially abnormal images for detailed human attention

\section{Process of Expert System Development}

Transferring expertise from human expert to the computer software system and then to the user involves four activities: knowledge acquisition, knowledge representation, knowledge inference and knowledge transfer [30]. Knowledge is acquired from experts and other documented sources and then represented or organized as rules or frames. These rules or frames are electronically stored as a body of knowledge or a knowledge base. The knowledge base forms one of the distinct components of an Expect system. The reasoning mechanism that draws conclusions from the knowledge attained in the knowledge base and the knowledge obtained from the user forms the other distinct components of the Expert System. This reasoning mechanism is known as the inference engine. The inference engine results in advice or recommendations for novices. When understood by the novice, possibly by accessing the explanation given by the Expert System, knowledge has been transferred to the user using user interface which is last component of an expert system [8].

\subsection{Knowledge Acquisition}

In designing expert systems, the process of eliciting information has been termed knowledge acquisition. Knowledge acquisition (elicitation) involves extracting problemsolving expertise from knowledge sources, which are usually domain experts [12]. In study of [31] knowledge acquisition defined as the process of extracting, structuring and organizing knowledge from several sources, usually human domain experts, so it can be used in a program. It involves the acquisition of knowledge from human experts, books, documents, sensors, or computer files.

Knowledge acquisition of is a major and critical phase in the development of an expert system. Knowledge acquisition is considered by many to be the most difficult and precarious stage in the knowledge engineering process. Knowledge acquisition has often been described as the bottleneck in knowledge based systems development today, thus, much theoretical and applied research is still being conducted in this area. The success of any expert system majorly depends on the quality, completeness, and accuracy of the information stored in the knowledge base [25], [5].

\subsection{Knowledge Representation}

Knowledge representation is the systematic means of encoding knowledge of the human expert in an appropriate medium. In expert systems development, a good solution 
depends on a good representation. For expert systems applications, the initial choice of a representation technique is particularly important. This is because the possible representation techniques are diverse and the forcing criterion for the choice is normally not clear at the outset of the project. The consequence of inadequate selection can be a major problem in the later stages of an Expert System development, if it is discovered that critical information cannot be encoded within the chosen representation technique [7].

\section{Coronary Artery Disease (CAD)}

Coronary Artery Disease (CAD), also known as Ischemic Heart Disease (IHD) or Coronary Heart Disease (CHD) is one of the deadliest diseases in the world. It occurs when plaque builds up inside the coronary arteries. These arteries supply heart muscle with oxygen-rich blood. Plaque is made up of fat, cholesterol, calcium, and other substances found in the blood. Over time, plaque hardens and narrows the arteries, reducing blood flow to heart muscle. Eventually, an area of plaque can rupture, causing a blood clot to form on the surface of the plaque. If the clot becomes large enough, it can mostly or completely block the flow of oxygen-rich blood to the part of the heart muscle fed by the artery [35].

It was estimated that nearly one half of all middle-aged men and one third of middleaged women in the United States have been affected with the disease [32]. CAD is the number one killer in the developed world, with over 7.4 million deaths attributed to Coronary Artery Disease in 2012 [34]. While, in Nigeria, Coronary Artery Disease has now become much familiar disease and the death rate is high due to the lack of awareness among the common people. According to the latest WHO data published in May 2014 CAD's deaths in Nigeria reached 53,836 or $2.82 \%$ of total deaths [34]. However, there are many literatures related to the knowledge acquisition and representation of expert system for diagnosis of coronary artery diseases.

\section{Literature Review}

In the study of [18] a fuzzy soft expert system to predict those patients who may suffer coronary artery disease using systolic blood pressure (SBP), low-density lipoprotein cholesterol LDL-C, maximum heart rate (MHR), blood sugar level (BS), old peak (OP) and age of patients was developed. The data used in the study was obtained from the Cardiac Unit, Department of Cardiology, Faculty of Medicine, Assiut University, Egypt of 200 patients containing 76 attributes. But only six of the attributes were considered because the authors of the study opinioned that only these six attributes are relevant to coronary artery disease which include blood pressure, cholesterol, maximum heart rate, blood sugar, old peak, age. However, in the study a small number of patient' records and limited numbers of the attributes were considered. The knowledge was acquired for the expert system using reviewing related records of the CAD's patients and accuracy of the system was not determined.

The study of [24] determined the accuracy of fuzzy rule-based classification that could noninvasively predict Coronary Artery Disease based on myocardial perfusion scan test and clinical-epidemiological variables. This was a cross-sectional study in which the characteristics, the results of myocardial perfusion scan (MPS), and coronary artery angiography of 115 patients, 62 (53.9\%) males, in Mazandaran Heart Center in the north of Iran have been collected. The system included 144 rules and the antecedent part of all rules has more than one parts. The coronary artery disease data used in the study contained 115 samples. The data was classified into four classes, namely, classes 1 (normal), 2 (stenosis in one single vessel), 3 (stenosis in two vessels), and 4 (stenosis in three vessels) which had 39, 35, 17, and 24 subjects, respectively. The accuracy in the fuzzy classification based on if-then rule was 92.8 percent if classification result was considered based on rule selection by expert, while it was 91.9 when classification result 
was obtained. However, the study only considered the results of myocardial perfusion scan (MPS), and coronary artery angiography of 115 patients without considering the other critical risks factors of the disease.

A fuzzy decision support system for the diagnosis of coronary artery disease based on evidence was developed in the study of [21]. The coronary artery disease data sets were collected from University California Irvine (UCI). The knowledge base of the system is taken by using rules extraction method based on Rough Set Theory. The rules then are selected and fuzzified based on information from discretization of numerical attributes. Fuzzy rules weight is proposed using the information from support of extracted rules. UCI heart disease data sets collected from U.S., Switzerland and Hungary, data from Ipoh Specialist Hospital Malaysia are used to verify the proposed system. The results show that the system is able to give the percentage of coronary artery blocking better than cardiologists and angiography. The results of the proposed system were verified and validated by three expert cardiologists and are considered to be more efficient and useful. However, the knowledge acquisition of the knowledge base of the system was based on evidence thus did not use data mining technique or other predictive techniques.

A rule-based Decision Support System was developed and implemented in study of [29] for the diagnosis of Coronary Artery Disease. The generation of the decision support system is realized automatically using a three stage methodology: (a) induction of a decision tree from a training set and extraction of a set of rules using id3 clarification algorithm (b) transformation of the set of rules into a fuzzy model and (c) optimization of the parameters of the fuzzy model. The system is evaluated using 199 subjects, each one characterized by 19 features, including demographic and history data, as well as laboratory examinations. Tenfold cross validation was employed and the average sensitivity and specificity obtained was $80 \%$ and $65 \%$ respectively. The approach of the study provides diagnosis based on easily acquired features and, since it is rule based, is able to provide interpretation for the decisions made. However, the study does not optimize the set of the rules generated before integrating them into the knowledge base.

In the study of [1], a fuzzy rule-based system (FRBS) was designed to serve as a decision support system for Coronary heart disease (CHD) diagnosis and it does not only considers the decision accuracy of the rules but also transparency at the same time. The data set used in the study was obtained from University California Irvine Repository of Machine Learning Databases. Originally, the data had 76 attributes, out of which only 13 attributes were selected for use. The selected attributes represent the clinical and noninvasive test results of 303 patients undergoing angiography. Removing the cases containing missing values, 270 cases were considered in study, out of which 120 cases are identified as patients with CHD while 150 cases are diagnosed as patients without CHD. However, the data used in the study originally had 76 attributes, out of which only 13 attributes were selected and used. The selected attributes represent the clinical and noninvasive test results of 303 patients undergoing angiography only.

Fuzzy Expert System for heart disease diagnosis using V.A. Medical Center, Long Beach and Cleveland Clinic Foundation database in the study of [2]. The system has 13 input fields and one output field. Input fields are chest pain type, blood pressure, cholesterol, resting blood sugar, maximum heart rate, resting electrocardiography (ECG), exercise, old peak (ST depression induced by exercise relative to rest), thallium scan, sex and age. The output field refers to the presence of heart disease in the patient. It is integer valued from 0 (no presence) to 4 (distinguish presence (values 1, 2, 3, 4)). However, the study used V.A. Medical Center, Long Beach and Cleveland Clinic Foundation database as a source of knowledge to the system without ascertain its relevance.

In the study of [9] screening system has been developed for the early detection of Coronary Artery Disease and it used only easily available clinical parameters and data obtained from very low cost laboratory tests. The risk classification has been done even before obtaining the next higher level information like ECG, angiogram, etc. The system 
was able to identify the risk group of patients at an early stage and consequently they will be guided for proper medication. However, for the acquisition of the knowledge for system of the study, a well-defined questionnaire was developed advised by domain experts and sample data were collected from the patients.

A fuzzy expert system and the imperialist competitive algorithm (ICA), to make coronary artery disease diagnosis by a non-invasive procedure were implemented in the study [36]. The imperialist competitive algorithm ICA was used to adjust the fuzzy membership functions. The proposed method has been evaluated with the Cleveland and Hungarian datasets. There are 597 records in total. There are 76 attributes in the datasets, of which all researches just use 14: 13 of them as inputs and 1 attribute as a result. The 13 input attributes include age, blood pressure, serum cholesterol, maximum heart rate, sex, chest pain type, fasting blood sugar, resting ECG, exercise-induced angina, old peak, slope, fluoroscopy, and thallium scan. The output variable is the angiography status. The advantage of the method, compared with others, is the interpretability. However, the study used fuzzy logic and imperialist competitive algorithm (ICA), instead of data mining technique and fuzzy logic.

A fuzzy rule-based system which concentrated only on accuracy and interpretability of the system was proposed by [15]. Fuzzy decision tree method was used based on fuzzy Relational Database Management System (RDBMS) and rules were generated $n$ based on C4.5 algorithm known as fuzzy rule generation system (FRGS) algorithm. However, the study concentrates only on the interpretability of the output of the system rather than accuracy of the generated rules and knowledge acquisition of the system fuzzy decision tree method based on fuzzy Relational Database Management System (RDBMS).

In the study [27] developed an expert system that provided a heart disease patient with background for suitable diagnosis and treatment (Especially Angina Pectoris and Myocardial infarction). The system was composed of four stages. The first stage was receiving the symptoms from the patient. The second stage was requesting from the patient to make some analysis and investigation to help the system to make a correct decision in the diagnosis. The third stage was doing diagnosis of patient according to information from patient (symptoms, analysis and investigation). The four stage was determining the name of appropriate medication or what should be done until the patient recovers (step therapy). The system was only able to give appropriate diagnosis and treatment for two heart diseases namely; angina pectoris and infarction. The programs used for diagnosis and system analysis include CLIPS and PROLOG. However, the study used validated interview and questionnaire for knowledge acquisition of the system and accuracy of the rules generated were not determined.

In study of [3] a web based Fuzzy Logic-based Expert System for the diagnosis of heart failure disease was developed. The system consists of a Knowledge Base (which is made up of a Database), a Fuzzy Logic component, a Fuzzy Inference Engine and a Decision Support Engine which comprises of cognitive and emotional filter as well as Tele medicine facilities. The system was implemented using Hypertext Preprocessor (PHP), JavaScript and Hypertext Mark-up Language (HTML) with My Structured Query Language (MySQL) as the Database Management System. However, the rules of the system were generated manually based on data that were selected of the heart failure patients and survey of some experts on heart failure disease at the State Specialist Hospital, Akure.

In the study of [33] an expert system for the diagnosis of the level of coronary heart disease by taking into account the problem of data imbalance was developed. The first stage of the research was preprocessing, which included resampled non-stratified random sampling (R), the synthetic minority over-sampling technique (SMOTE), clean data out of range attribute (COR), and remove duplicate (RD). The second step was the sharing of data for training and testing using a k-fold cross-validation model and training multiclass classification by the K-star algorithm. The third step was performance evaluation. The 
proposed system was evaluated using the performance parameters of sensitivity, specificity, positive prediction value (PPV), negative prediction value (NPV), area under the curve (AUC) and F-measure. Results: The results showed that the proposed system provides an average performance with sensitivity of $80.1 \%$, specificity of $95 \%$, PPV of $80.1 \%$, NPV of $95 \%$, AUC of $87.5 \%$, and Fmeasure of $80.1 \%$. Performance of the system without consideration of data imbalance provide showed sensitivity of $53.1 \%$, specificity of $88,3 \%$, PPV of $53.1 \%$, NPV of $88.3 \%$, AUC of $70.7 \%$, and F-measure of $53.1 \%$. But the study used the patient data from the UCI repository. The coronary heart disease data was obtained from Roberto Detrano, $\mathrm{MD}, \mathrm{PhD}$, which is a collection of data from the VA Medical Center. Coronary heart disease was distributed into five levels or types, namely, healthy, sick-low, sick-medium, sick-high, and sick-serious. The data comprised 303 instances of data and 14 parameters, with one parameter as an indication of the level of heart disease on a scale of 0-4. However, the data used in the study was collected from Roberto Detrano, MD, PhD, which he also collected from the VA Medical Center without validation.

A coronary artery disease fuzzy expert system for microarray data classification using a novel Genetic Swarm Algorithm, has been proposed in the study of [10] for obtaining near rule set and membership function tuning. The convergence of genetic swarm algorithm and their classification accuracy were improved by using the advanced and problem specific genetic operators. The major disadvantages in the study is that, the probability distribution of genes is not here to compute the mutual information and the rule set is not tuned properly due to rounding off problems.

The neural networks ensemble method has been proposed in the study of [23] for the effective diagnosis of the heart disease. This ensemble method was able to create new models by combining the posterior probabilities or the predicted values from multiple predecessor models. Here the SAS base software 9.1.3 method was used for diagnosing the heart disease. The major disadvantage of the study is that, the artificial neural network with a back propagation algorithm is used to learn by changing the connection weights.

The fuzzy neural network and k-fold cross validation has been proposed in the study of [13] for a hybrid system of diagnosis of diabetes and heart diseases. The back propagation algorithm was used to train a fuzzy network. The classification accuracies of the datasets were obtained by the k-fold cross validation. The major disadvantage of study is that; the missing values are not handled properly.

The different data mining techniques such as neural networks, decision trees and naive bayes has been proposed by [6] for the study of heart disease prediction system. The multi-layer perceptron neural networks were used to map the input data onto the output data. However, the system was not developed to ascertain its validity.

The adaptive neuro fuzzy inference system and Advanced fuzzy resolution mechanism has been proposed in the study of [26] to diagnose the heart disease. The Advanced fuzzy resolution mechanism was designed with predictive value and if then rules to diagnosis the heart disease. The accuracy of this system is comparatively low, which need to be further improved.

An evolutionary fuzzy expert system is proposed for the diagnosis of the Coronary Artery Disease (CAD) based on Cleveland clinic foundation datasets for heart diseases in the study of [20]. The decision tree was used to select the most significant attributes and the output is converted into crisp if-then rules. The crisp sets of rules are transformed into the fuzzy rules and these rules constitute the fuzzy rule base. Genetic Algorithm (GA) is used to tune the fuzzy membership functions and the optimized of membership functions using GA helps to achieve better accuracy. The accuracy of this system is comparatively low, which need to be further improved.

In the work of [16] a computer intelligent based approach for the diagnosis of heart diseases was had developed. Apriori, Predictive Apriori and Tertius were the three different rule mining algorithms used to present rule extraction experiment on heart 
disease data and showed as efficiency algorithm for diagnosis task. Cleveland dataset, a publicly available dataset and widely popular with data mining researchers, have been used for diagnosis because of the privacy problem related to medical data set. Generally diagnosis were costly, time consuming and likely to suffer from error. The analyzed information available on sick and healthy individuals indicted that females have less chance of coronary heart disease than males. Heart disease for both men and women was existed only in the presence of exercise-induced angina and factors such as chest pain were asymptotic. The resting ECG for men and women was different. The risk factors of resting ECG for women were being either normal or hyper and slope being flat. And only a single rule expressing resting ECG being hyper was an important factor for men. Slope being up, number of colored vessels being zero and old peak being less than or equal to 0.56 indicated after comparing the healthy status of men and women. However, the system was not developed and implemented to ascertain its validity.

In the work of [22] classification method based on preprocessing the data first with Principal Component Analysis (PCA) had applied and then applying differential evolution classifier to the diagnosis of heart disease. This method was applied here for predictind diagnosis from clinical data sets with chief complaint of chest pain using classical Electronic Medical Record (EMR), heart data sets which contains demographic properties, clinical symptoms, clinical findings, laboratory test results specific ElectroCardioGraphy (ECG), results pertaining to angina and coronary infarction. Individually they computed results for four different heart data sets and also the results for the case when all data sets were combined together. It was to demonstrate and assess the proposed classification approach. They were considered that the main factor resulting in the good classification accuracy in studied cases was the application of an effective global optimizer, differential evolution, for fitting the classification model instead of local optimization based approaches. With diagnosis of heart disease, they found the data preprocessing with PCA. Here higher classification accuracy can be achieved than without preprocessing. The result indicated that prepossessing the data before classification might not only help with the curse of increasing data dimensionality, but also provide a further improvement in classification accuracy. They managed to classify the Switzerland data set with $94.5 \% \pm 0.4 \%$ mean accuracy. They were combined the data sets and achieved the mean accuracy of $82 \% \pm 0.5 \%$. Here, the classification accuracy yielded by the proposed approach was outperformed when compared with the other corresponding results of several classifiers. However, the system was not developed and implemented to ascertain its validity.

In work [4] a weighted fuzzy rule-based Clinical Decision Support System (CDSS) for computer-aided diagnosis of the heart disease was developed. The proposed CDSS for risk prediction of the heart patients contains two steps such as: generation of weighted fuzzy rules and developing of a fuzzy rule-based decision support system. Here, data preprocessing was applied on the heart disease data set for removing the noisy information and to find missing values. After that using the frequent attribute categories, the deviation range and relevant attributes were computed in this method. According to the deviation range, the attributes were selected if any deviation exists or not and also the deviation range was used to construct the decision rules. Those decision rules were scanned in the learning database to find out its frequency. As per its frequency the weight age was calculated for every decision rule obtained and by the help of fuzzy membership function, the weighted fuzzy rules were obtained. The automatic procedure to generate the fuzzy rules was an advantage of the proposed system and the weighted procedure introduced in the proposed work was additional advantage for effective learning of the fuzzy system. However, the accuracy of the system was not determined. 


\section{Discussion}

There have been so many studies by scholars to address the drawbacks of knowledge acquisition and representation for system expert of early prediction of coronary artery disease but it was very clear that these studies have some drawbacks, which necessitated to have further study to come up with a framework or approach for Knowledge acquisition and presentation for expert system of early prediction and detection of Coronary Artery Disease (CAD) to addressed the identified drawbacks in the various studies so far reviewed which include the following:-

i. Most of the studies used validated interview and questionnaire and review of the related documents or material for knowledge acquisition of the system

ii. The accuracy of the rules generated in most of the studies reviewed was not determined.

iii. The accuracy of the most of the systems developed in the studies reviewed was not determined.

iv. None of the study reviewed handle the missing values properly.

v. None of the study used Nigerian based data to generate the rules for the expert system of CAD.

vi. The studies reviewed so far none of them optimize the set of the generated rules before integrating them into the knowledge base.

\section{Conclusion}

In this study, a detailed review of previous studies has been carried out by various researchers in the field of development of expert system for diagnosis of coronary artery disease, their drawbacks and strengths were identified and presented. The study concluded that, there is a need for further study to come up with a multi layered framework or approach for knowledge acquisition and presentation for expert system of early prediction and detection of Coronary Artery Disease (CAD) so as to address those identified drawbacks in this study.

\section{References}

[1] L. Adel, N. A. Raja, Z. Roziati and B. Awang, "Design of a Fuzzy-based Decision Support System for Coronary Heart Disease Diagnosis", J Med Syst, @ S Springer, (2012).

[2] A. Ali and N. Mehdi, "A Fuzzy Expert System for Heart Disease Diagnosis", Proceedings of the International Multi Conference of Engineers and Computer Scientists, vol. 1, (2010), pp. 17-19.

[3] O. C. Akinyokun, G. B. Iwasokun, S. A. Arekete and R. W. Samuel, "Fuzzy logic-drive expert system for the diagnosis of heart failure disease", Artificial Intelligence Research, vol. 4, no. 1, (2015).

[4] P. K. Anooj, "Clinical decision support system: Risk level prediction of heart disease using weighted fuzzy rules", Journal of Computer and Information Sciences, vol. 24, (2012), pp. 27-40.

[5] T. Byrd, K. Cossick and R. Zmud, "A synthesis of research on requirements analysis and knowledge acquisition techniques”, MIS Quarterly, vol. 16, no. 1, (1992), pp. 117-138.

[6] S. D. Chaitrali and S. A. Sulabha, "Improved Study of Heart Disease Prediction System using Data Mining Classification Techniques", International Journal of Computer Applications, vol. 47, no. 7, (2012), pp. 0975-888.

[7] S. Chen, "A knowledge acquisition scheme for ruled-based systems", Proceedings IEEE Region 10 Conference on Computer, Communication, Control and Power Engineering, vol. 2, (1993), pp. 621-625.

[8] E. De Kock, "Expert Systems and knowledge acquisition", Lecture Note. University of Pretoria etd., (2003).

[9] P. Debabrata, K. M. Mandana, P. Sarbajit, S. Debranjan and C. Chandan, "Fuzzy expert system approach for coronary artery disease screening using clinical parameters", Elsevier, Journal of Knowledge-Based Systems, vol. 36, (2012), pp. 162-174.

[10] K. P. Ganesh, A. V. Aruldoss, P. Renukadevi and D. Devaraj, "Design of fuzzy expert system for microarray data classification using a novel Genetic Swarm Algorithm", Expert Systems with Applications, vol. 39, pp. 1811-1821. 
[11] A. Goodall, "Expert Systems (Computer science)", Learned Information, University of California, (1985).

[12] R. Hoffman, "The problem of extracting knowledge of experts from the prospective of experimental psychology", AI Magazine, vol. 8, (1987), pp. 53-64.

[13] K. Humar and A. Novruz, "Design of a Hybrid System for the Diabetes and Heart Diseases Expert Systems with Applications", vol. 35, (2008), pp. 82-89.

[14] G. Hwang, "Knowledge elicitation and integration from multiple experts", Journal of Information Science and Engineering, vol. 10, (1994), pp. 99-109.

[15] M. Idris, A. Pervez, J. A. Tariq and S. Z. Syed, "Fuzzy Rule Based Classification for Heart Dataset using Fuzzy Decision Tree Algorithm based on Fuzzy RDBMS World”, Applied Sciences Journal, vol. 28, no. 9, (2013), pp. 1331-1335.

[16] N. Jesmin and I. Tasadduq, "Association rule mining to detect factors which contribute to heart disease in males and females", Journal of Expert Systems with Applications, vol. 40, (2013), pp. 1086-1093.

[17] L. J. Muhammad, S. Sani, Y. Atomsa, M. M. Yusuf, T. A. Elrufai, I. A. Mohammed and A. M. Nuhu, "Using Decision Tree Data Mining Algorithm to Predict Causes of Road Traffic Accidents, its Prone Locations and Time along Kano -Wudil Highway", International Journal of Database Theory and Application, vol. 10, no. 11, (2017), pp. 197-208.

[18] H. Nasruddin, R. S. Osama, M. K. Ahmed and A. G. Mohammed, "Fuzzy Soft Expert System in Prediction of Coronary Artery Disease", International Journal of Fuzzy Systems, (2016).

[19] National Institutes of Heart, Lung, and Blood Institute (NHLBI), "The Fifteenth Report of the Joint National Committee on Prevent, Detection, Evaluation, and Treatment of High Blood Pressure”, 015, 5256, (2013).

[20] Y. D. Niranjana and S. Anto, "An Evolutionary-Fuzzy Expert System for the Diagnosis of Coronary Artery Disease", International Journal of Advanced Research in Computer Engineering \& Technology (IJARCET), vol. 3, no. 4, (2014).

[21] A. S. Noor, P. A. Venkatachalam and F. H. Ahmad, "Diagnosis of Coronary Artery Disease Using Artificial Intelligence Based Decision Support System", Proceedings of the International Conference on Man-Machine Systems (ICoMMS) 11, Batu Berringhi, Penang, Malaysia, (2009).

[22] L. Pasi and L. Jouni, "A Classification Method Based on Principal Component Analysis and Differential Evolution Algorithm Applied for Prediction Diagnosis from Clinical EMR Heart Data Sets", Journal of Computer Intelligence in Optimization Adaption Learning and Optimization, vol. 7, (2010), pp. 263283.

[23] D. Resul, T. Ibrahim and S. Abdulkadir, "Effective diagnosis of heart disease through neural networks ensembles", Expert Systems with Applications, vol. 36, (2009), pp. 7675-7680.

[24] A. M. Reza, M. A. Seyed, B. Somayeh and G. Ali, "Fuzzy Rule-Based Classification System for Assessing Coronary Artery Disease", Hindawi Publishing Corporation Computational and Mathematical Methods in Medicine, (2015).

[25] F. Rook and J. Croghan, "The knowledge acquisition activity matrix: a systems engineering conceptual framework", IEEE Transaction on Systems, Man \& Cybernetics, vol. 19, no. 3, (1989), pp. 586-597.

[26] A. V. Senthil Kumar, "Diagnosis of heart disease using advanced fuzzy resolution mechanism", Journal of Artificial Intelligence, pp. 1-9.

[27] R. A. Soltan, M. Z. Rashad and B. El-Desouky, "Diagnosis of Some Diseases in 'Medicine via computerized Experts", International Journal of Computer Science \& Information Technology (IJCSIT), vol. 5, no. 5, (2013).

[28] S. S. Smita, S. Sushil and M. S. Ali, "Fuzzy Expert Systems (FES) for Medical Diagnosis", International Journal of Computer Applications (0975 - 8887), vol. 63, no. 11.

[29] M. G. Tsipouras, T. P. Exarchos, D. I. Fotiadis, A. Kotsia, A. Naka and L. K. Michalis, "A Decision Support System for the Diagnosis of Coronary Artery Disease", Proceedings of the IEEE Symposium on Computer-Based Medical Systems, (2008).

[30] E. Turban and E. Jay, "Decision Support Systems and Expert Systems", 6 ${ }^{\text {th }}$ Edition, Copyright 2001, Prentice Hall, Upper Saddle River, NJ, (2001).

[31] D. A. Waterman, "A guide to expert systems. Reading", Mass: Addison-Wesley Publishing, (1985).

[32] P. W. F. Wilson and P. S. Douglas, "Epidemiology of coronary heart disease. UpToDate", Retrieved from http://www.uptodate.com/contents/epidemiology--of--coronary--heart-disease, (2015)

[33] W. M. Wiharto, K. Hari and H. Herianto, "Intelligence System for Diagnosis Level of Coronary Heart Disease with K-Star Algorithm", Healthcare Informatics Research, vol. 22, no. 1, (2016), pp. 30-38.

[34] World Health Organization (WHO)., 2015, Cardiovascular diseases, factsheet\#317.Retrieved from http://www.who.int/mediacentre/factsheets/fs317/en/ last accessed (2017) January 16.

[35] N. D. Wong, "Epidemiological studies of CHD and the evolution of preventive cardiology", Nature reviews, Cardiology, vol. 11, no. 5, (2014), pp. 276-89.

[36] M. Zahra and S. A. Mohammad, "CADICA: Diagnosis of Coronary Artery Disease Using the Imperialist Competitive Algorithm", Journal of Computing Science and Engineering, vol. 8, no. 2, (2014), pp. 87-93. 
International Journal of $u$ - and e- Service, Science and Technology

Vol. 11, No.3 (2018) 